\title{
APLICAÇÃO DE INDICADOR DE ACEITABILIDADE E SUA EFICIÊNCIA NA ANÁLISE DOS FATORES ASSOCIADOS À SATISFAÇÃO SOBRE DIFERENTES PÚBLICOS EM UNIDADES DE ALIMENTAÇÃO E NUTRIÇÃO
}

\section{ACCEPTABILITY INDICATOR EFFICIENCY ANAL YSIS AND CHARACTERIZATION OF FACTORS ASSOCIATED WITH DIVERGED PUBLIC INSIDE DIFFERENT FOOD AND NUTRITION UNITS}

\author{
Bruna Dalpizol ${ }^{1}$, Adriana Hernandes Martins ${ }^{2^{*}}$ \\ ${ }^{1}$ Acadêmica do curso de Nutrição do Centro Universitário Fundação Assis Gurgacz - FAG. ${ }^{2}$ Docente \\ do Curso de Nutrição do Centro Universitário Fundação Assis Gurgacz - FAG. Especialista em \\ Gestão da Qualidade em Alimentos- UNICAMP \\ *Autor correspondente: adrihernandesm@gmail.com, https://orcid.org/0000-0001-9718-5846
}

DOI: $10.35984 / f j h . v 2 i 1.180$

\section{RESUMO}

O estudo do tipo qualitativo avalia os parâmetros determinantes na satisfação global de refeições em quatro Unidades de Alimentação e Nutrição situadas em Cascavel, Paraná, Brasil, a correlação com o grau de apetite, sintomas físicos e gastrointestinais. Na obtenção dos dados, utilizou-se como base o Questionário de Satisfação em Cuidados Intensivos de Serviço Alimentar do Paciente - ACHFPS - (Acute Care Hospital Foodservice Patient Satisfaction Questionnaire), desenvolvido por Capra et al. (2005). A amostra apresentou 220 indivíduos com idade média de 44,90 \pm 19,65 anos, de ambos os sexos e estado de saúde variado. A satisfação global foi satisfatória na maioria das categorias, mas insatisfatória quanto à variedade de frutas e à quantidade da porção ofertada. Não se obteve significância de aceitabilidade entre os gêneros, mas, quando subdivididos por estado de saúde, observouse maior aceitação em indivíduos com patologias, assim como com relação ao apetite e aos sintomas físicos e gastrointestinais.

Palavras-chave: Nutrição. Questionário. Aceitabilidade.

\begin{abstract}
The aim of this qualitative study was to evaluate the parameters for the global menu satisfaction into four different Food and Nutrition Units situated in Cascavel, Paraná, Brazil, its correlation with the appetite, gastrointestinal and physical symptoms. For data collection was took the Acute Care Hospital Foodservice Patient Satisfaction Questionnaire - ACHFPS - as a basis, created for Capra et al., (2005). The sample submitted 220 individuals with an average age of 44,90 $\pm 19,65$ years old, both sexes and with a health status variation. The global satisfaction was excellent for the majority indicators, but showed unsatisfaction for fruits variety and food portion quantity indicators. There was no significance between genders and acceptability, but when divided between health status, was possible to observe that pathology group was significant satisfied than the healthy group, the same was found for the appetite scale and for gastrointestinal and physical symptoms.
\end{abstract}

Keywords: Nutrition. Questionnaire. Acceptability.

\section{INTRODUÇÃO}

Os macronutrientes e micronutrientes presentes nos alimentos são a base da nutrição humana. Assim, o profissional de nutrição em Unidades de Alimentação e 
Nutrição, tanto em empresas quanto em hospitais, desempenha um papel fundamental de mediador na educação nutricional, na prevenção, no tratamento à saúde e contra a desnutrição de uma população (GARCIA et al., 2006). Portanto, uma Unidade de Alimentação e Nutrição constitui-se o veículo fornecedor de refeições nutricionalmente balanceadas, seguindo as normas dietéticas e higiênico-sanitárias junto aos padrões financeiros de cada organização (ABREU, 2013).

O Programa de Alimentação ao Trabalhador (PAT) enfatiza o auxílio das organizações na melhora das condições nutricionais dos indivíduos, contribuindo de $30 \%$ a $40 \%$ do Valor Energético Total diário nas principais refeições (almoço, jantar e ceia). Todavia, sabe-se que a preferência e a escolha alimentar de uma população são intrínsecas a cada cultura, ao nível escolar, à idade e ao sexo. Por isso, é importante ao profissional de nutrição conhecer o público ao qual aplica suas estratégias nutricionais para estar em equilíbrio com as escolhas alimentares de cada grupo cultural (ALVARENGA et al., 2015). Obtendo-se informações para um cardápio bem planejado e bem aceito por meio de indicadores de satisfação, é possível dentro de uma organização potencializar o capital humano pela melhora do rendimento e saúde do pessoal (OECD, 2018) e ainda ser utilizada como um objeto ativo e condizente com os objetivos firmados e promovidos pela ONU na Agenda 2030 para o Desenvolvimento Sustentável (2015), que determinam saúde e bem-estar no ambiente empresarial, elementos importantes para o crescimento global. No âmbito hospitalar, essa ferramenta pode ser contribuinte para formulação de cardápios que auxiliem na dietoterapia do paciente, na ingestão desejada de macronutrientes e micronutrientes, na melhora do estado nutricional, na diminuição do tempo de internamento, na redução de custos desnecessários, na redução significativa de restos ingestas e na melhora na satisfação dos serviços (ALLARD et al., 2015; ROLLINS e DOBAK, 2018; MCCRAY et al., 2018).

Diante disso, indicadores de satisfação são ferramentas com informações significativas sobre o público que se deseja trabalhar (MCCRAY et al., 2018), contudo, a coleta de informações em inquéritos deve ser rápida, de fácil entendimento a todo tipo de público, causar menos interrupções possíveis ao entrevistado e adquirir o máximo de informações, não somente identificando a aceitação global, mas também identificando os aspectos específicos das refeições que contribuem para satisfação como um todo (HANNAN-JONES e CAPRA, 2016).

O questionário de satisfação em cuidados intensivos de serviço alimentar do paciente ACHFPS (Acute Care Hospital Foodservice Patient Satisfaction Questionnaire), desenvolvido por Capra et al. (2005), é uma ferramenta válida, confiável e sensível. Aplicado em hospitais do mundo inteiro, prova evidências diante de quatro estruturas: qualidade do alimento, qualidade do serviço alimentar, serviço de funcionários e ambiente físico. Além da satisfação global, avalia também aspectos importantes como temperatura, grau de escolha e variedade dos alimentos, o que contribuem para a qualidade das refeições e dos serviços (HANNAN-JONES e CAPRA, 2016).

Diante desse contexto, o objetivo deste estudo foi analisar a eficiência na aplicação dessa ferramenta não somente em comensais hospitalizados como realizado em estudos anteriores, mas também com públicos de Unidades de Alimentação e Nutrição (UAN) distintas, englobando indivíduos adultos e em idade avançada, tanto saudáveis quanto acometidos enfermos. Além de trazer a análise do grau de satisfação global diante da amostra total de um público heterogêneo, também se buscou identificar a influência da satisfação global de acordo com possíveis 
sintomas gástricos, físicos e grau de apetite diante de um perfil demográfico, de gênero e de estado de saúde.

\section{METODOLOGIA}

O estudo do tipo qualitativo foi desenvolvido em quatro UANs distintas na cidade de Cascavel, Paraná, entre os períodos de julho de 2019 a novembro de 2019. A pesquisa foi aprovada pelo comitê de Ética em Pesquisa do Centro Universitário Assis Gurgacz de Cascavel, Paraná, sob o parecer de número 3.457.728.

O estudo englobou ao todo uma população de 220 indivíduos em quatro UANs na cidade de Cascavel - PR, localizada ao sul Brasil. A primeira UAN hospitalar era composta com indivíduos em estado pós-operatório, em estado de saúde estável, dislipidêmicos, hipertensos e diabéticos principalmente. A segunda UAN institucional era voltada ao público em idade avançada, tanto de estado de saúde estável e sem patologias, quanto dislipidêmicos, hipertensos e diabéticos principalmente. A terceira UAN institucional com característica de refeitório contava com indivíduos de estado de saúde estável e sem patologias. Na quarta e última UAN institucional com característica de refeitório, havia indivíduos com estado de saúde estável e sem patologias.

Para aplicação do estudo com as três UAN's em âmbito organizacional, os critérios de seleção foram: indivíduos que estivessem frequentando o local há pelo menos um dia consecutivo, faixa etária acima de 18 anos de idade, conscientes e aptos para responder ao inquérito individualmente, assim como dispostos a participar do estudo. Os critérios de exclusão determinaram indivíduos que se alimentaram em um período de um dia sem ser em dias consecutivos e de faixa etária menor de 18 anos de idade. Os participantes selecionados foram dispostos em posições variadas e confortáveis de forma que permanecessem um ao lado do outro, para então serem direcionados a assinar ao Termo de Consentimento Livre e Esclarecido (TCLE). Posteriormente, foram submetidos a uma entrevista semiestruturada baseada no Questionário de Satisfação em Cuidados Intensivos de Serviço Alimentar do Paciente ACHFPSQ (Acute Care Hospital Foodservice Patient Satisfaction Questionnaire), de Capra et al. (2005), a fim de medir a satisfação e a aceitação dos cardápios elaborados e aplicados em UAN's institucionais.

Para aplicação do estudo em UAN em âmbito hospitalar, os critérios de seleção foram indivíduos internados pelo Sistema Único de Saúde (SUS), assim como pacientes internados em leitos particulares e convênios, em período de internamento de pelo menos 24 horas, conscientes e aptos a responderem ao inquérito, estado patológico de leve a moderado, incluindo estado pós-operatório, puérperas, de ambos os sexos, e faixa etária acima de 18 anos de idade. Os critérios de exclusão foram indivíduos internados por período menor de 24 horas, inconscientes, acometidos por doenças mentais, como doença de Alzheimer e outras doenças de grau grave, pacientes em jejum, em dietas enterais e parenterais, acompanhantes, crianças recém-nascidas, faixa etária até 18 anos de idade e funcionários do local. Os pacientes selecionados foram primeiramente convidados a participar do estudo por livre e espontânea vontade e direcionados a assinar ao termo de Consentimento Livre e Esclarecido (TCLE), seguindo pela aplicação do questionário em forma de entrevista direta baseada no Questionário de Satisfação em Cuidados Intensivos de Serviço Alimentar do Paciente ACHFPSQ (Acute Care Hospital Foodservice Patient Satisfaction Questionnaire) de Capra et al. (2005), a fim de medir a satisfação e a 
aceitação das dietas hospitalares. O inquérito foi aplicado igualmente nas quatro UAN's. Com relação aos indivíduos hospitalizados e aos de idade avançada com dificuldades em responder individualmente, prestou-se ajuda.

$O$ inquérito desenvolvido baseado no questionário traduzido do inglês para o português brasileiro - ACHFPSQ (Acute Care Hospital Foodservice Patient Satisfaction Questionnaire) de Capra et al. (2005) - foi constituído por 15 questões a serem categorizadas em uma escala de Likert com cinco níveis de satisfação de "ruim" a " excelente", acrescidas de mais três afirmações, "regular", "bom", "muito bom" e perguntas sobre a satisfação global, como saciedade, expectativas referentes ao alimento, aspectos visuais e sensoriais, textura, temperatura, diversidade, cordialidade dos funcionários e ambiente. A afirmação existente quanto ao apetite foi categorizada por três níveis: "Mais baixo que o normal", "normal" e "mais alto que o normal". Para a afirmação existente quanto aos sintomas gastrointestinais, as categorias de resposta foram: "Nunca", "raramente", "às vezes", "frequentemente" e "sempre". As questões individuais de satisfação global foram posteriormente classificadas de 1 a 5, sendo que a pontuação 1 representa o resultado menos positivo e a pontuação 5 representa o resultado mais positivo. Em adicional, foram elaboradas categorizações quanto ao gênero - feminino ou masculino -; idade e patologia aferida, se assim obtivesse.

Os resultados foram expressos por meio da estatística descritiva e analisados quanto à sua normalidade dos resíduos pelo teste de Shapiro-Wilk. Para comparar a variável idade entre os grupos, foram empregados os testes de Mann-Whitney-U e de Kruskal-Wallis post-hoc Dunn's. O teste de Qui-quadrado foi adotado para associar o grau de apetite, de aceitação do cardápio e dos sintomas gastrointestinais entre os gêneros e a condição patológica. O programa $R$ Version 1.1.453 - (C) 2009-2018, Inc. foi utilizado para realizar os testes. Valores considerados significativos foram de $5 \%$.

\section{RESULTADOS E DISCUSSÃO}

A amostra deste estudo apresentou 220 indivíduos com idade média de 44,90 $\pm 19,65$ anos. O sexo feminino apresentou uma frequência de $60,5 \%$, sendo dominante na amostra, e o masculino $39,5 \%$.

$\underline{\text { Tabela 1. Valores absolutos e relativos entre as categorias de gênero, local e patologia da amostra }}$

\begin{tabular}{ccc}
\hline Categorias & $\mathbf{n ~ ( 2 2 0 )}$ & $\%$ \\
\hline Gênero & & \\
Masculino & 87 & 39,5 \\
Feminino & 133 & 60,5 \\
Local & & \\
Idosos & 55 & 25,0 \\
Trabalhadores Convencionais A & 55 & 25,0 \\
Trabalhadores Convencionais B & 55 & 25,0 \\
Hospitalizados & 55 & 25,0 \\
Patologia & & \\
Sim & 66 & 30,0 \\
Não & 154 & 70,0 \\
\hline
\end{tabular}

As quatro UAN's apresentaram frequências relativas iguais de $25,0 \%$ para o público de idosos, trabalhadores convencionais $A$, trabalhadores convencionais $B$ e 
público hospitalizado. Observou-se que 30,0\% da amostra tinha alguma patologia, dentre elas: diabetes mellitus do tipo dois, hipertensão arterial e estado pósoperatório. A associação de duas ou mais dessas patologias também pode ser constatada (Tabela 2), sendo que "puérperas" foram devidamente alocadas ao grupo sem alguma patologia, conforme indica a Tabela 1.

Observou-se na amostra uma dominância de $70 \%$ de indivíduos saudáveis e $30 \%$ indivíduos com patologias, possibilitando a amostra ser subdividida nesses dois grupos citados. Os indivíduos saudáveis tiveram uma predominância nas UAN's voltadas ao público de trabalhadores convencionais $A$ e $B$, como também ao público de idosos. Os indivíduos com patologias foram predominantes tanto na UAN hospitalar quanto ao público de idosos, justificando as patologias com maiores frequências, como pós-operatório, hipertensão, pós-operatório mais hipertensão e diabetes tipo dois mais hipertensão, assim representadas pela tabela 2.

Tabela 2. Valores de frequência absoluta e relativa em relação às categorias de patologia da amostra

\begin{tabular}{ccc}
\hline Patologia & $\mathbf{n ~ ( 2 2 0 )}$ & $\%$ \\
\hline Nenhuma & 147 & 66,8 \\
PO & 21 & 9,5 \\
HA & 17 & 7,7 \\
PO / HA & 10 & 4,5 \\
Diabetes / HA & 7 & 3,2 \\
Puérperas & 7 & 3,2 \\
Diabetes & 5 & 2,3 \\
PO / HA / Diabetes & 4 & 1,8 \\
PO / Diabetes & 2 & 0,9
\end{tabular}

Legenda: $\mathrm{PO}=$ pós-operatório. $\mathrm{HA}=$ hipertensão arterial.

Quanto aos resultados da aceitação global total da amostra $(\mathrm{N}=220)$, diagnosticou-se a predominância para a categoria "excelente", sendo que os maiores valores observados nesse indicador foram os de cordialidade dos funcionários, de saciedade ao finalizar cada refeição e do ambiente. Isso corrobora o estudo realizado por Salamiah et al. (2015) em comensais de um restaurante institucional e por Herezais et al. (2018) em hospitais privados e públicos, em que ambos encontraram em seus resultados que, devido aos serviços de funcionários e ao ambiente, 0 indicador de satisfação global chegou a atingir significativamente 68,2\% e acima de $90 \%$, respectivamente. Esses dados demonstram que os serviços de atendimento, tanto oferecidos por copeiras em hospitais quanto por funcionários responsáveis pela distribuição dos alimentos, são contribuintes significantes para a preferência e a satisfação do indivíduo no ambiente e na adesão do cardápio.

Em seguida, a categoria "bom" apresentou o segundo maior percentual na satisfação global, seguindo pela categoria "muito bom", sendo possível notar, desse modo, que alguns indicadores ainda necessitam de grandes melhorias para chegar à excelência. Os maiores scores obtidos foram os indicadores de horários para oferta, sabor e textura dos alimentos, aparência das preparações e variedade/combinação de vegetais com o restante da preparação. A baixa satisfação quanto ao horário de oferta das refeições foi observada por Herezais et al. (2018), em que alguns comensais dentro do ambiente hospitalar justificaram sua insatisfação devido aos horários serem distintos dos convencionais. Aminuddin et al. (2018), ao aplicarem o questionário ACHFPSQ em pacientes hospitalizados, diagnosticaram baixa satisfação referente aos indicadores de variedade das preparações, de aparência, de 
variedade/combinação dos vegetais e de sabor e textura das preparações, relatando também a insatisfação de comensais hospitalizados quanto a esses indicadores. Os resultados do presente estudo podem ser comparados aos encontrados por Cavalcante e Baratto (2018), que, apesar de ter sido aplicado questionário distinto, os resultados obtidos foram semelhantes quanto ao nível de satisfação. Esse resultou em regular quanto ao sabor e à variedade do cardápio entre comensais de um restaurante institucional, muito próximo ao obtido neste presente estudo, sendo possível enfatizar a necessidade de adaptação das preparações e da aparência dos alimentos.

A expectativa quanto ao alimento a ser ofertado dentro do ambiente hospitalar, de trabalho ou em restaurantes acaba sendo baixa, especialmente quanto ao sabor, à qualidade e à aparência das louças (PRICE et al., 2016). No decorrente estudo, uma das maiores frequências encontradas para a categoria "regular" foi ao indicador de exceptiva quanto ao alimento a ser ofertado, indicando a insatisfação. Comparandose esses indicadores com as análises realizadas por Price et al. (2016), justifica-se que os alimentos consumidos em hospitais e em restaurantes não são os mesmos consumidos convencionalmente.

As categorias "regular" e "ruim" apresentaram os menores percentuais diante da satisfação global. Por mais baixos que sejam esses percentuais, foi possível obter e analisar quais indicadores necessitam de grandes melhorias para chegar à excelência. As maiores frequências obtidas foram para a variedade das frutas e a quantidade da porção ofertada; nesse último, a categoria "ruim" se sobressaiu à categoria "regular", indicando maior insatisfação. A insatisfação quanto à porção ofertada confronta com o resultado obtido neste estudo para o indicador de saciedade, que apresentou um dos maiores percentuais de satisfação. Segundo pacientes em internamento, a porção ofertada acaba sendo em quantidade excedente, consequentemente, havendo desperdícios. Os desperdícios acabam sendo comuns mundialmente, uma metanálise realizada por Williams e Walton (2011), em 32 países incluindo o Brasil, foi possível notar um percentual de $6 \%$ a $65 \%$ em restos ingestas, visto que são indicadores de vários fatores e, segundo Aminuddin et al. (2018), mais estudos são necessários para compreender esse mecanismo.

O grau de apetite é uma motivação para ingestão de determinados alimentos e pode variar no momento da refeição, podendo ser alto, normal ou muito baixo (CAPRA et al., 2005). O baixo apetite dentre o público de idosos no ambiente hospitalar acaba sendo esperado e, muitas vezes, vem acompanhado de sintomas como refluxos, náuseas e enjoos, justificando que o próprio ambiente acaba sendo um determinante, medicamentos eventuais e de rotina ou mesmo a injúria aferida (HOPE et al., 2017). No presente estudo, o grau de apetite "normal" se sobressaiu em $76,8 \%$, ao passo que houve uma prevalência para a categoria "nunca" quanto aos sintomas gastrointestinais referentes a enjoos/mal-estar/azias, seguindo para a categoria "às vezes".

Sintomas físicos como tontura/fraqueza/sonolência podem ser recorrentes de acordo com a combinação de alimentos ingestos, por exemplo, o excesso de carboidratos provoca um aumento momentâneo do hormônio insulina, resultando em sintomas físicos característicos (RIIS et al., 2019). Para trabalhadores convencionais, esses sintomas necessitam ser minimizados, evitando-se a fadiga no período pósprandial. Os resultados obtidos por este estudo apontam uma prevalência da categoria "nunca", distribuídos entre as categorias "às vezes" e "raramente", "sempre" e "frequente" foram as categorias com menores frequências; porém, ainda presentes, 
se comparadas aos sintomas gastrointestinais sem nenhuma frequência absoluta para a categoria "sempre".

Tabela 3. Valores de frequência absoluta e relativa da amostra em relação ao grau de apetite, aos níveis de aceitação do cardápio e aos sintomas físicos

\begin{tabular}{|c|c|c|c|c|c|}
\hline Categorias & n (220) & $\%$ & Categorias & n (220) & $\%$ \\
\hline \multicolumn{3}{|l|}{ Grau de apetite } & \multicolumn{3}{|c|}{ Sabor e textura dos alimentos } \\
\hline Mais baixo que o normal & 26 & 11,8 & Ruim & 4 & 1,8 \\
\hline Normal & 169 & 76,8 & Regular & 26 & 11,8 \\
\hline \multirow[t]{2}{*}{ Mais alto que o normal } & 25 & 11,4 & Bom & 67 & 30,5 \\
\hline & & & Muito bom & 30 & 13,6 \\
\hline \multicolumn{3}{|c|}{ Expectativa quanto ao alimento } & Excelente & 93 & 42,3 \\
\hline Ruim & 5 & 2,3 & \multicolumn{3}{|c|}{ Saciedade ao finalizar } \\
\hline Regular & 25 & 11,4 & Ruim & 2 & 0,9 \\
\hline Bom & 57 & 25,9 & Regular & 24 & 10,9 \\
\hline Muito bom & 44 & 20,0 & Bom & 63 & 28,6 \\
\hline Excelente & 89 & 40,5 & Muito bom & 21 & 9,5 \\
\hline \multicolumn{3}{|l|}{ Aparência da refeição } & Excelente & 110 & 50,0 \\
\hline Ruim & 3 & 1,4 & \multicolumn{3}{|l|}{ Horário de oferta } \\
\hline Regular & 18 & 8,2 & Ruim & 9 & 4,1 \\
\hline Bom & 75 & 34,1 & Regular & 15 & 6,8 \\
\hline Muito bom & 34 & 15,5 & Bom & 71 & 32,3 \\
\hline Excelente & 90 & 40,9 & Muito bom & 20 & 9,1 \\
\hline \multicolumn{3}{|c|}{ Quantidade da porção ofertada } & Excelente & 105 & 47,7 \\
\hline Ruim & 19 & 8,6 & \multicolumn{3}{|c|}{ Cordialidade dos funcionários } \\
\hline Regular & 13 & 5,9 & Ruim & 10 & 4,5 \\
\hline Bom & 61 & 27,7 & Regular & 15 & 6,8 \\
\hline Muito bom & 28 & 12,7 & Bom & 47 & 21,4 \\
\hline Excelente & 99 & 45,0 & Muito bom & 24 & 10,9 \\
\hline \multicolumn{3}{|l|}{ Cozimento dos alimentos } & Excelente & 124 & 56,4 \\
\hline Ruim & 6 & 2,7 & \multicolumn{3}{|l|}{ Ambiente } \\
\hline Regular & 24 & 10,9 & Ruim & 6 & 2,7 \\
\hline Bom & 56 & 25,5 & Regular & 13 & 5,9 \\
\hline Muito bom & 29 & 13,2 & Bom & 59 & 26,8 \\
\hline Excelente & 105 & 47,7 & Muito bom & 33 & 15,0 \\
\hline \multicolumn{3}{|c|}{ Temperatura dos alimentos } & Excelente & 109 & 49,5 \\
\hline Ruim & 4 & 1,8 & \multicolumn{3}{|c|}{ Enjoo / Mal estar / Queimação } \\
\hline Regular & 13 & 5,9 & Nunca & 153 & 69,5 \\
\hline Bom & 63 & 28,6 & Raramente & 26 & 11,8 \\
\hline Muito bom & 41 & 18,6 & Às vezes & 34 & 15,5 \\
\hline Excelente & 99 & 45,0 & Frequente & 7 & 3,2 \\
\hline \multicolumn{3}{|l|}{ Variedade de frutas } & Sempre & 0 & 0,0 \\
\hline Ruim & 26 & 11,8 & \multicolumn{3}{|c|}{ Sonolência / Tontura / Fraqueza } \\
\hline Regular & 26 & 11,8 & Nunca & 145 & 65,9 \\
\hline Bom & 51 & 23,2 & Raramente & 29 & 13,2 \\
\hline Muito bom & 38 & 17,3 & Às vezes & 29 & 13,2 \\
\hline Excelente & 79 & 35,9 & Frequente & 8 & 3,6 \\
\hline \multicolumn{3}{|c|}{ Variedade de vegetais e combinação } & Sempre & 9 & 4,1 \\
\hline Ruim & 7 & 3,2 & & & \\
\hline Regular & 23 & 10,5 & & & \\
\hline Bom & 67 & 30,5 & & & \\
\hline Muito bom & 25 & 11,4 & & & \\
\hline Excelente & 98 & 44,5 & & & \\
\hline
\end{tabular}

Ao analisar a Tabela 4, observou-se associação entre gênero e os locais de coleta dos dados $(p=0,001)$. Foram verificadas maiores frequências para o sexo feminino ao público de idosos, e o sexo masculino para o público de trabalhadores 
convencionais. Para os locais de trabalhadores convencionais B e hospitalizados, a distribuição entre os gêneros foi igual.

Com relação ao gênero e à patologia, as frequências foram iguais $(p=0,278)$, ou seja, tanto o sexo masculino quanto o feminino apresentaram frequências iguais em ter ou não alguma patologia.

Tabela 4. Análise das variáveis local e patologia em relação ao gênero

\begin{tabular}{ccc}
\hline & \multicolumn{3}{c}{ Gênero } \\
\cline { 2 - 3 } Variáveis & $\mathrm{X}^{2}(\mathrm{GL})$ & $\mathrm{p}$ \\
\hline Local & $15,267(3)$ & $\mathbf{0 , 0 0 1}$ \\
Patologia & $1,173(1)$ & 0,278 \\
\hline
\end{tabular}

$\mathrm{X}^{2}=$ valor do teste do qui-quadrado. $\mathrm{GL}=$ graus de liberdade.

A Tabela 5 apresenta os valores de significância entre gênero e patologia em relação aos níveis de satisfação global, ao apetite e aos possíveis sintomas físicos e gastrointestinais.

Tabela 5. Associação do grau de apetite, aceitabilidade da dieta e sintomas gastrointestinais em relação ao gênero e à patologia

\begin{tabular}{lccccc}
\hline & Variáveis & \multicolumn{2}{c}{ Gênero } & \multicolumn{2}{c}{ Patologia } \\
& $X^{2}(G L)$ & $p$ & $X^{2}(G L)$ & $p$ \\
\hline Apetite & $0,956(2)$ & 0,620 & $13,165(2)$ & $<0,001^{*}$ \\
Grau de apetite & & & & &
\end{tabular}

\section{Aceitabilidade da dieta}

Expectativa quanto ao alimento

$8,486(4) \quad 0,075 \quad 42,181(4) \quad<0,001^{*}$

Aparência da refeição

$5,187(4) \quad 0,268 \quad 55,423(4) \quad<0,001^{*}$

Quantidade da porção ofertada

$1,320(4) \quad 0,857$

$31,335(4)<0,001^{*}$

Cozimento dos alimentos

$2,103(4) \quad 0,716$

$68,055(4) \quad<0,001^{*}$

Temperatura dos alimentos

$1,779(4) \quad 0,776$

$43,387(4) \quad<0,001^{*}$

Variedade das frutas

$5,931(4) \quad 0,204$

$39,040(4) \quad<0,001^{*}$

Variedade de vegetais e combinação

14,662 (4) $\quad 0,005^{*}$

$35,558(4)$

$<0,001^{*}$

Sabor e textura dos alimentos

$2,101(4) \quad 0,717$

$67,715(4)$

$<0,001^{*}$

Saciedade ao finalizar

$8,783(4) \quad 0,066$

$51,978(4)$

$<0,001^{*}$

Horário de oferta

$7,082(5) \quad 0,214$

$54,120(5)$

$<0,001^{*}$

Cordialidade dos funcionários

$8,113(5) \quad 0,150$

$44,521(5)$

$<0,001^{*}$

Ambiente

$3,311(4) \quad 0,507$

$49,901(4)$

$<0,001^{*}$

\section{Sintomas físicos e gastrointestinais}

Enjoo / mal estar / queimação

$\begin{array}{llll}0,950(3) & 0,813 & 17,957(3) & <0,001^{*} \\ 2,274(4) & 0,685 & 23,315(4) & <0,001^{*}\end{array}$

Sonolência / tontura / fraqueza

do. $\mathrm{GL}=$ graus de liberdade.

Conforme Ncube e Letsoalo (2019), hospitais devem considerar o gênero um fator determinante na experiência hospitalar e no serviço de alimentação, visto que os homens mostram maior satisfação para todos os indicadores do questionário ACHFPS 
do que as mulheres, reforçando o estudo realizado por Naglaa et al. (2017), no qual o gênero masculino dentro do ambiente hospitalar mostrou-se significativamente mais satisfeito do que o gênero feminino. Já Mccray et al. (2018) não encontraram nenhuma diferença significativa relacionada aos gêneros e aos níveis de satisfação global que fora classificada em "Bom" e "Muito bom". Associando isso ao atual estudo, foram constatadas apenas diferenças entre os gêneros para o indicador de variedade de vegetais e sua combinação com o restante da refeição $(p=0,005)$, verificando-se menores frequências para a categoria "Muito bom" diante do sexo feminino e maiores frequências para o sexo masculino, ou seja, o sexo feminino apresentou menor aceitação apenas nesse indicador. Para os demais indicadores, não foram observadas frequências desajustadas. É possível afirmar, portanto, que a aceitabilidade das refeições não difere significativamente diante do sexo feminino e masculino, sendo positivos os níveis de satisfação para ambos os gêneros.

Ao associar o grau de apetite com o gênero, não foi verificado significância $(p=0,620)$, observando uma distribuição de frequências normais para as categorias baixo, normal e médio apetite entre o sexo feminino e masculino. Quanto aos sintomas físicos e gastrointestinais, também não foram obtidas diferenças significativas entre os gêneros.

Segundo McCray et al. (2018), é comum o decréscimo de apetite no indivíduo acometido por alguma patologia. Em indivíduos hospitalizados, por exemplo, esse sintoma pode decair ainda mais devido às questões psicológicas. Em idosos acometidos pelo estado pós-operatório e com doenças respiratórias, o risco de mortalidade mostrou-se ser alto, visto que o seu baixo apetite e o baixo consumo de alimentos eram decorrentes do uso de medicamentos para o tratamento, da falta de mobilidade, do ambiente, da falta de funcionários animados ou mesmo do sentimento de depressão (HOPE et al., 2017). Os mesmos autores enfatizam que os pacientes não priorizam os alimentos durante o período de internamento, pois acreditam serem as medicações os agentes mais importantes do que a alimentação em si.

Neste presente estudo, no que diz respeito à patologia e ao grau de apetite, verificou-se associação significativa $(p<0,001)$. Para os indivíduos que têm patologias, verificou-se maiores frequências para a categoria de "Baixo" apetite. Já para os indivíduos que não têm patologias, foram observadas maiores frequências para a categoria de apetite "Normal". Isso se torna coerente com os resultados encontrados por outros autores citados, mostrando que as patologias adquiridas, como o diabetes mellitus do tipo dois, hipertensão, estado pós-operatório e a associação de uma ou mais dessas patologias, interferem no grau de apetite do indivíduo, sendo determinantes para a ingesta alimentar, a recuperação do estado de saúde e a aceitação da refeição. Machado et al. (2013) enfatizam que esse sintoma pode ser persuadido pela qualidade, pela apresentação e pelas características sensoriais das refeições ofertadas, tais como sabor, apresentação, aparência, aroma, diversidade do cardápio, temperatura e textura. Ainda, se distribuídas em seis refeições diárias, a satisfação no tocante à aparência e ao sabor do alimento pelo paciente é otimizada, com concomitante aumento da sua ingesta (DIJXHOORN et al., 2017).

Além do baixo apetite constatado por Hope et al. (2017), os indivíduos acometidos por patologias respiratórias e estado pós-operatório descreveram experiências como náuseas e refluxos enquanto estavam hospitalizados, sendo significativos quanto à ingesta de alimentos e à aceitação da dieta. Neste atual estudo, os sintomas gastrointestinais como enjoo, mal-estar e queimação também apresentaram associação significativa em relação à patologia. Verificou-se que os 
indivíduos com alguma patologia apresentaram maiores frequências para categoria "Nunca". Já para os indivíduos que não têm alguma patologia, observou-se maiores frequências para a categoria "Raramente", diferenciando-se dos resultados obtidos por Hope et al. (2017). Indivíduos em estado pós-operatório, com patologias adquiridas, como diabetes mellitus do tipo dois, hipertensão e associação de duas ou mais destas patologias, estão mais propensos aos cuidados alimentares intensivos ou ao uso de medicamentos específicos devido ao estado de saúde, resultando em um fator positivo contra a manifestação dos sintomas físicos e gastrointestinais negativos.

Ao analisar os indicadores de satisfação global, verificou-se que os indivíduos com alguma patologia apresentaram maiores frequências para a categoria "Excelente" em todos os 13 indicadores. Os indivíduos que não têm alguma patologia apresentaram maiores frequências para as categorias "Bom", "Muito bom" e "Regular", nessa respectiva ordem.

Quando foi analisada apenas a categoria "Ruim", foram verificadas maiores frequências para indivíduos com alguma patologia diante do indicador "aparência da refeição" e "sabor e textura dos alimentos". Maciel et al. (2019) conseguiu elevar a satisfação do cardápio para 85\% após intervenções gastronômicas nas principais refeições, sem modificação da aparência, mas aprimorando o sabor e a textura dos alimentos, pela mudança no corte da proteína e mudança de coloração de determinados alimentos, pela adição de legumes ou maior tempo e pelo método de cocção. Enfatiza-se que o sabor, a textura e a apresentação dos alimentos melhoram aceitabilidade e reduzem os índices de resto ingestas. Já os indivíduos sem alguma patologia, apresentaram maiores frequências de rejeição para o indicador "horário de oferta da refeição".

De modo geral, o grupo patológico apresentou o grau de aceitabilidade mais elevado em relação ao grupo não patológico devido à apresentação de maiores frequências para excelência em todos os indicadores de aceitação global. Esses achados podem ser comparados aos resultados obtidos por Souza et al. (2018), que constataram alta satisfação e aceitabilidade das dietas especificas orais ofertadas a indivíduos hipertensos e diabéticos mellitus do tipo dois, assim como os resultados de Casado e Barbosa (2015), que, ao investigarem a aceitação das dietas hipossódicas, verificaram que $81,8 \%$ dos pacientes qualificaram as dietas como ótima (3\%), muito boa $(9,1 \%)$ e boa $(69,7 \%)$. Mostrando que os conhecimentos adquiridos e repassados pelo profissional de nutrição em relação á técnicas de cocção, de preparo e de prépreparo dos alimentos estão sendo aderidos diante de uma população com patologias adquiridas que necessitam de conhecimento para obtenção de mudanças de hábitos. Se observa também, que a consciência nas questões relacionadas a saúde e alimentação estão aumentando positivamente emergindo numa reeducação alimentar permanente.

Neste estudou notou-se algumas variáveis, quanto á consciência de todos os indivíduos da amostra e seu estado atual de saúde. Muitos indicaram possuir nenhuma patologia presente no momento, porém sabe-se que a falta e diagnostico pode ser alta ou pela falta de acompanhamento médico, pela não presença de sintomas negativos e consequentemente o indivíduo não ter ciência do seu próprio estado de saúde.

\section{CONCLUSÃO}

De acordo com os resultados obtidos pela aplicação do questionário de satisfação em cuidados intensivos de serviço alimentar do paciente ACHFPS (Acute 
Care Hospital Foodservice Patient Satisfaction Questionnaire), desenvolvido por Capra et al. (2005), foi possível determinar o grau de satisfação de um público heterogêneo, não somente com indivíduos hospitalizados, como realizado por estudos anteriores. $O$ instrumento utilizado mostrou ser uma ferramenta rápida, confiável, sensível e de fácil entendimento, provando evidências diante da qualidade do alimento, do serviço alimentar, serviço de funcionários, ambiente físico, grau de apetite e o diagnóstico de sintomas físicos e gastrointestinais decorrentes da ingesta. Ao profissional de nutrição, informações de suma importância emergiram, visto que é de responsabilidade do nutricionista a elaboração de cardápios respeitando todos os níveis culturais, todas as idades, gêneros e estado de saúde.

A satisfação global mostrou ser positiva para seus 13 indicadores, enfatizando que as refeições ofertadas estão de acordo com as características de cada UAN e ao público em geral. O maior indicador na aceitabilidade foi quanto à cordialidade de funcionários, à saciedade ao finalizar cada refeição e ao ambiente. A insatisfação obtida foi quanto à variedade de frutas e à quantidade da porção ofertada. Os níveis de aceitação global não mostraram diferença significativa entre os gêneros, porém, quando subdivididos por patologias, observou-se que indivíduos acometidos por diabetes mellitus do tipo dois, hipertensão, estado pós-operatório ou a junção dessas duas ou mais patologias, teve significância a aceitação das refeições, sendo indivíduos com patologias mais satisfeitos em relação aos indivíduos saudáveis, assim como na manifestação quanto aos sintomas físicos e gastrointestinais.

Para que progressivamente os níveis de satisfação cresçam, é necessária rotineiramente a aplicação de questionários de aceitabilidade, como o ACHFPS, pois, ao diagnosticar fatores que indicam a insatisfação dos comensais, é possível desenvolver e reaplicar outros questionários mais específicos ou então partir para tomada de medidas corretivas dentro do centro distribuidor de refeições, as Unidades de Alimentação e Nutrição. Estudos detalhando a característica demográfica, sexo e estado de saúde de uma população, são necessários para implementar as pesquisas de satisfação e consequentemente para obtenção de resultados mais precisos na interpretação do perfil de comensais e consequentemente na aderência qualitativa das refeições, contrabalanceando com as características e padrões pertencentes a cada instituição.

\section{REFERÊNCIAS}

ABREU, E. S; SPINELLI S.; NEUMANN, M.G.; SOUZA, A.M. Gestão de Unidades de Alimentação e Nutrição: Um Modo De Fazer. 5a․ Ed., São Paulo: E.d Metha LTDA., p.35-42, 2013.

ALLARD, J. P., KELLER, H., TETERINA, A.; JEEJEEBHOY, K. N.; LAPORTE, M., DUERKSEN; LOU, W. Factors associated with nutritional decline in hospitalised medical and surgical patients admitted for 7 days or more: A prospective cohort study. British Journal of Nutrition. V. 114, n. 10, p. 1612-1622, 2015.

ALVARENGA, M.; KORITAR, P.; ORGSS. Determinantes de escolhas e consumo, nutrição comportamental. 2ª . ed. Barueri: Manoele, p. 33-35, 2015.

AMINUDDIN, N. F.; VIJAYAKUMARAN, R. K.; ABDUL RAZAK, S. Patient satisfaction with hospital foodservice and its impact on plate waste in public 
hospitals in east Malaysia. Hospital Practices and Research. V. 3, n. 3, p. 90-97, 2018.

CAPRA, S.; WRIGHT, O.; SARDIE, M.; BAUER, J.; ASKEW, D. The acute hospital foodservice patient satisfaction questionnaire: the development of a valid and reliable tool to measure patient satisfaction with acute care hospital foodservices. Food service Research International. V.16 e.2, p. 14, 2005.

CASADO, V. D. M.; BARBOSA, L. S. Aceitação de dieta hipossódica e estado nutricional de pacientes internados em hospital público de Goiânia. $\mathbf{O}$ mundo da saúde. V. 39, n. 2, p. 188-194, 2015.

CAVALCANTE, J. M.; BARATTO, I. Pesquisa de satisfação em um restaurante universitário no Sudoeste do Paraná-PR. RBONE-Revista brasileira de obesidade, nutrição e emagrecimento. V. 11, n. 68, p. 661-666, 2018.

DIJXHOORN, D. N.; WANTEN, G. J. A.; VAN DEN BERG, M. G. A. OR24: A novel in-hospital meal service improves protein and energy intake, a prospective study. Clinical Nutrition. 36: S10, 2017.

GARCIA, R. W. D. A dieta hospitalar na perspectiva dos sujeitos envolvidos em sua produção e em seu planejamento. Revista Nutrição, Campinas. V. 19, n. 2, p. 129144, 2006.

HANNAN-JONES, M.; CAPRA, S. Prevalence of diet-related risk factors for chronic disease in male prisoners in a high secure prison. European journal of clinical nutrition. V. 70. e. 2 p. 212, 2016.

HEREZAIS, A. C.; BARATTO, I.; GNOATTO, F. Avaliação da satisfação das refeições servidas em hospitais do município de Pato Branco-PR. RBONE-Revista brasileira de obesidade, nutrição e emagrecimento. V. 12, n. 72, p. 479-485, 2018.

HOPE, K.; FERGUSON, M.; REIDLINGER, D. P.; AGARWAL, E. "I don't eat when I'm sick": Older people's food and mealtime experiences in hospital. Maturitas. V. 97, p. 6-13, 2017.

MACHADO, A. D; OLIVON, E. V.; MATIAS, A. C. G.; de ABREU, E. S. Avaliação do almoço oferecido a pacientes oncológicos e transplantados pediátricos pelo método AQPC. Revista de ciências médicas e biológicas. V. 12, n. 3, p. 3323-336, 2013.

MACIEL, I. J. L.; DE SOUZA, V. C. S.; ANDRADE, N. C.; DA CONCEIÇÃO, L. D. K. M.; DOS SANTOS MORAIS, B. H. Intervenções gastronômicas e análise da aceitabilidade em um restaurante universitário na cidade de Belém-PA. DEMETRA: Alimentação, nutrição e saúde. V. 14, n. 38974, 2019.

MCCRAY. S; MAUNDER K.; KRIKOWA R.; MACKENZIE-SHALDERS K. Room service improves nutritional intake and increases patient satisfaction while decreasing food waste and cost. Journal of the academy of nutrition and dietetics. V. 118, $\mathrm{n}$. 2, p. 284-293, 2018. ISSN 2212-2672. 
NAGLAA A.; EL-SHERBINY.; IBRAHIM E. H.; HEWEDI M. M. Patients satisfaction with delivered food services in Fayoum hospitals. EC Nutrition. V. 9, e. 2, p. 94-104, 2017.

NCUBE, L.; LETSOALO, M. Foodservice quality in South African hospitals: patient experiences. International journal of health care quality assurance. V. 32 n. 3, p. 599-610, 2019.

PARIS. OECD Economic Surveys: Brazil 2018, OECD Publishing. 2018.

ONU. Organização das Nações Unidas. Transformando Nosso Mundo: A Agenda 2030 para o Desenvolvimento Sustentável, 2015.

PRICE, S.; HARTWELL, H.; HEMINGWAY, A.; CHAPLEO, C. Workplace foodservice; perception of quality and trust. Appetite. V. 97, p.169-175, 2016.

BRASIL. Programa de Alimentação do Trabalhador. Portaria interministerial n. 66, de 25 de agosto de 2006. Diário Oficial da União de 28 de agosto de 2006.

RIIS, S.; MØLLER, AB.; DOLLERUP, O.; HØFFNER, L.; JESSEN, N.; MADSEN, $\mathrm{K}$. Acute and sustained effects of a periodized carbohydrate intake using the sleep-low model in endurance-trained males. Scandinavian journal medical sciences sports. V. 0, n. 0, p. $115,2019$.

ROLLINS C., DOBAK S. Creating a great patient experience: improving care with food and nutrition services. Journal of the academy of nutrition and dietetics. V. 118, $\mathrm{n}$. 5, p. 805-808, 2018.

SALAMIAH A. J.; SALLEH M. R.; NORZUWANA S.; C.T. CHIK, MOHD FAEEZ SAIFUL BAKHTIAR. Hospitality and Tourism 2015: Proceedings of HTC 2015. CRC Press, 2015.

SOUZA, M. C. G. C.; MENDES, A. L. D. R. F.; SANTOS, G. C. M. D.; BRITO, F. C. R.; MORAIS, V. D. D.; SILVA, F. R. D. Estado nutricional e aceitação da dieta por pacientes cardiopatas. Motricidade. V. 14, n.1, p. 217-225, 2018.

WILLIAMS P.; WALTON K. Plate waste in hospitals and strategies for change. ESpen, the European Journal of Clinical and Nutrition Metabolism. V. 6, n. 6, p. 235-241, 2011. 